\title{
ITHANET: Information and database community portal for
}

\section{haemoglobinopathies} \author{
Petros Kountouris $^{1,}$, , Coralea Stephanou ${ }^{1}$, Celeste Bento ${ }^{2}$, Pavlos Fanis ${ }^{1}$, Jacques Elion ${ }^{3}$,
} Raj S Ramesar ${ }^{4}$, Bin Alwi Zilfalil ${ }^{5}$, Helen M Robinson ${ }^{6}$, Joanne Traeger-Synodinos ${ }^{7}$, Human Variome Project Global Globin 2020 Challenge, Carsten W Lederer ${ }^{1}$, and Marina Kleanthous ${ }^{1}$

${ }^{1}$ Molecular Genetics Thalassaemia, The Cyprus Institute of Neurology and Genetics, Nicosia, Cyprus

${ }^{2}$ Haematology Department, Centro Hospitalar e Universitário de Coimbra, Coimbra, Portugal

${ }^{3}$ Paris Diderot University Medical School, Paris, France

${ }^{4}$ Division of Human Genetics, Department of Pathology, University of Cape Town, Cape Town, South Africa

${ }^{5}$ Universiti Sains Malaysia, Kelantan, Malaysia

${ }^{6}$ Human Variome Project International Coordinating Office, Melbourne, Australia

${ }^{7}$ Medical Genetics, National and Kapodistrian University of Athens, Athens, Greece

* Corresponding author (The Cyprus Institute of Neurology and Genetics, P.O. Box 23462, 1683

Nicosia, CYPRUS, Tel: (+357) 22392 657, Fax: (+357) 22392 615, E-mail: petrosk@cing.ac.cy)

\section{ABSTRACT}

Haemoglobinopathies are the commonest monogenic diseases, with millions of carriers and patients worldwide. Online resources for haemoglobinopathies are largely divided into specialised sites catering for patients, researchers and clinicians separately. However, the severity, ubiquity and surprising genetic complexity of the haemoglobinopathies call for an integrated website to serve as a free and comprehensive repository and tool for patients, scientists and health professionals alike. This paper presents the ITHANET community portal, an expanding resource for clinicians and researchers dealing with haemoglobinopathies. It integrates information on news, events, publications, clinical trials and haemoglobinopathyrelated organisations and experts and, most importantly, databases of variations, epidemiology and diagnostic and clinical data. Specifically, ITHANET provides annotation for 2690 haemoglobinopathy-related variations, epidemiological data for more than 180 countries and information for more than 600 HPLC diagnostic reports. The ITHANET portal accepts and incorporates contributions to its content by local experts from any country in the world and is freely available for the public at http://www.ithanet.eu. 
36 Haemoglobinopathies are the commonest monogenic diseases in the world, posing a major public health problem worldwide. It is estimated that around $5.2 \%$ of the world's population carry a potentially pathogenic globin gene and that, annually, over 330 thousand newborns bear a serious haemoglobinopathy [1]. Haemoglobinopathies comprise the thalassaemias, the sickle-cell disorders $(\mathrm{SCD})$, haemoglobin $(\mathrm{Hb}) \mathrm{E}, \mathrm{Hb} \mathrm{C}$ and other, rarer disorders and are mainly caused by mutations in the two globin-gene clusters, namely the $\alpha$-globin locus (Chromosome: 16, Accession: NG_000006) and the $\beta$-globin locus (Chromosome: 11, Accession: NG_000007), which can cause defects in the structure of $\mathrm{Hb}$ or reduced synthesis of globin chains and of $\mathrm{Hb}$ within the red blood cells. To date, more than 2000 diseasecausing mutations have been reported, affecting different levels of gene regulation and expression [2].

Through resistance of carriers to the malaria parasite, haemoglobinopathies have a high prevalence in former malaria regions in the Mediterranean, the Middle East, South-East Asia and Sub-Saharan Africa [3-5]. However, demographic events, such as migration from highprevalence areas and the consequent intermixing of populations, have contributed to the

51 spread of haemoglobinopathies worldwide [6-8] and, consequently, their prevalence is rising in non-endemic regions, such as Northern and Western Europe and North America, posing a major challenge for researchers and health professionals. In addition, the lack of high-quality epidemiological data, the high variation of disease prevalence in different ethnic groups and in different regions within the same country, as well as general underestimates of haemoglobinopathy incidence, particularly in low- and middle-income countries, are serious impediments to appropriate policy making $[9,10]$.

58 Recent advances in biotechnology, particularly the emergence of high-throughput sequencing 59 technologies, as well as recent increased research interest in the field of rare diseases, which 60 affect fewer that one in 2000 population-wide, have led to an explosion in the amount of 61 genetic, clinical and diagnostic information. In the field of haemoglobinopathies, the commonest rare disease in the world, online resources are largely divided into specialised sites catering for patients, researchers and clinicians separately, while available information 
64 has been fragmented into different databases, mainly variation-centric and genotype-

65 phenotype databases. More specifically, relevant information can be found in genome-wide resources, such as dbSNP [11], OMIM [12], ClinVar [13] and HGMD [14], but the level of detailed annotation varies, particularly for rare, yet clinically significant, variations [15]. In addition, locus-specific databases, such as HbVar [16] and several databases based on the Leiden Open Variation Database (LOVD) software [17,18], have been successful in the past, but their curation has been a challenging task. Therefore, there is an urgent need for an

71 integrated resource that will bring together all available haemoglobinopathy-related information and will set up a community-based framework for the continuous and consistent collection and integration of high-quality data.

Herein, we present the ITHANET community portal, an expanding resource for clinicians and researchers dealing with haemoglobinopathies, that

(a) integrates the latest haemoglobinopathy-related information, including news, events, publications and clinical trials, (b) provides information about the worldwide community of organisations and experts working on haemoglobinopathies, and (c) develops, and maintains databases of variations, epidemiological, diagnostic and clinical data. The ITHANET portal was initially the result of

80 a multinational Euro-Mediterranean project [19] and has progressed to becoming an 81 expanding community resource in collaboration with the Global Globin 2020 (GG2020)

82 Challenge by the Human Variome Project (HVP). GG2020 is now officially linked to the 83 ITHANET portal after review of all known existing databases in the field [20] and with 84 additional partnership through a shared ITHANET-GG2020 Expert Panel application for haemoglobinopathy-related variant classification under the Clinical Genome (ClinGen) Resource. The ITHANET portal welcomes contributions to its content and acknowledges all 87 contributions in a specifically designed section. ITHANET is free and available at http://www.ithanet.eu.

\section{Data Structure and Management}

91 The ITHANET portal is freely accessible online for viewing, searching and administrating as 
93 (http://www.php.net) based on the "Joomla!" content management system

94 (http://www.joomla.org) and uses the jQuery JavaScript library (http://www.jquery.com), as

95 well as packages jQuery-UI (http://www.jqueryui.com), DataTables

96 (http://www.datatables.net), HighCharts and HighMaps (http://www.highcharts.com), to

97 enhance the presentation of the data. The interface does not require the installation of

98 additional plugins, such as Flash and Microsoft Silverlight, and, thus, works natively across

99 all modern web browsers and the majority of mobile web browsers. All data available in

100 ITHANET are stored and organised in a relational database using MySQL

101 (http://www.mysql.com), an open-source relational database management system widely

102 utilised in database design in bioinformatics and biomedical informatics. ITHANET is hosted

103 by the Cyprus Institute of Neurology and Genetics (http://www.cing.ac.cy) using Apache 2

104 HTTP Server (http://www.apache.org).

\section{Data Collection and Database Curation}

106 A key component for the creation of a public knowledge base is the efficient collection,

107 validation and annotation of relevant information. ITHANET uses a combination of automatic

108 and manual curation to update and enrich the portal's content. Weekly updates on scientific

109 literature are automatically received from PubMed using the following search query:

110 "thalassemia [tiab] OR thalassaemia [tiab] OR hemoglobin [tiab] OR haemoglobin [tiab]

111 OR sickle-cell [tiab] OR hemoglobinopathies [tiab] OR haemoglobinopathies [tiab]".

112 Subsequently, the references are manually filtered to find relevant information. Importantly,

113 ITHANET is an active partner in and beneficiary of primary research data from several

114 projects on haemoglobin disorders, while additional information is retrieved through

115 subscriptions to scientific societies and organisations. In addition, ITHANET accepts and

116 incorporates contributions to its content by local experts from any country in the world and

117 acknowledges their contribution eponymously in affected content pages of databases

118 IthaGenes and IthaMaps and on a specifically designed page ("Contributors and Curators").

\section{RESULTS}

120 As illustrated in Figure 1, existing content on the ITHANET portal can be divided into four

121 main categories: (a) provision of the latest information related to haemoglobinopathies (e.g. 
123 experts working on haemoglobinopathies, and (c) databases and computational tools.

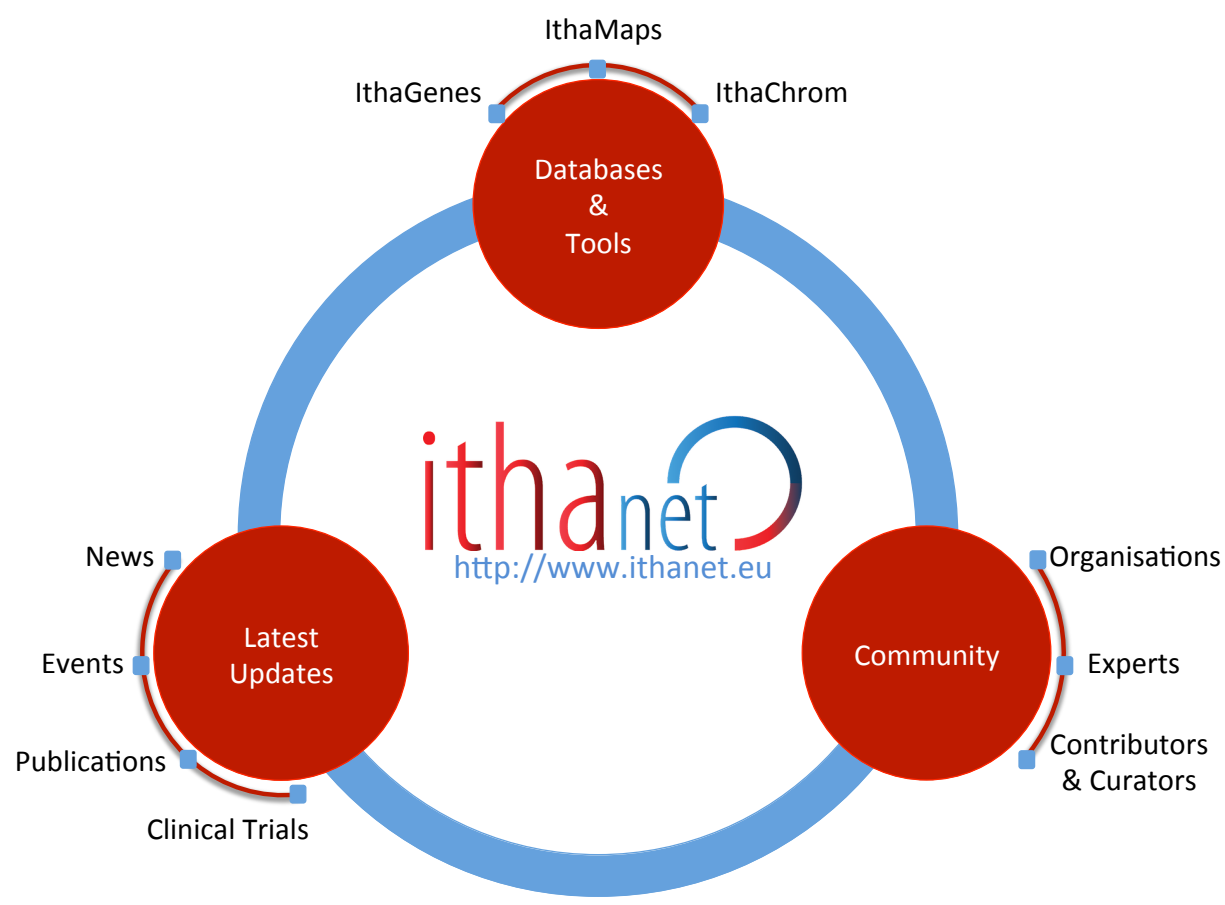

Figure 1: An overview of the content on the ITHANET portal

\section{Latest Updates}

128 Through its role as a community resource, the ITHANET portal collects and provides the

129 most recent information about haemoglobinopathies and closely related topics from the fields

130 such as human genetics and genomics. Specifically, recent news, such as new research grants,

131 significant discoveries, press releases and clinical trials, are provided in the "News" section,

132 while searchable lists of past and future scientific events, recent publications and clinical 133 trials are provided in sections "Events", "Publications" and "Clinical Trials", respectively.

134 Information on scientific events is retrieved through subscriptions to numerous scientific 135 organisations/societies, patient organisations and research networks, while information on 136 publications and clinical trials is retrieved on a weekly basis from NCBI PubMed and 137 clinicaltrials.gov, respectively. In October 2017, manually curated entries of these ITHANET 138 sections comprised 149 news items, 271 past or future events, 3993 publications and 411 
139 clinical trials. Moreover, registered subscribers are informed about the ITHANET updates

140 through a regular electronic newsletter.

141 Community

142 International collaboration and synergies are critical for tackling the burden of rare diseases,

143 in which the number of patients and, consequently, experts specialising on specific diseases is

144 small. In an effort to map institutions and scientists with expertise in haemoglobinopathies

145 and to facilitate collaboration, ITHANET provides a searchable list of organisations related to

146 haemoglobinopathies, with detailed description, contact details and affiliated experts and,

147 similarly, a searchable list of experts working on different areas of haemoglobinopathies with

148 detailed information about contact details, research interests and affiliations and, importantly,

149 possible contributions to ITHANET content. In October 2017, ITHANET stored information

150 about 147 organisations and 174 experts, of whom 29 have also directly contributed to its

151 content in all major sections of the portal, i.e. IthaGenes, IthaMaps and IthaChrom. Such

152 contributions are critical to the accuracy, expansion and growing success of the portal and are

153 therefore acknowledged in a specific section ("Contributors and Curators").

\section{Databases and Tools}

\section{IthaGenes}

156 IthaGenes is a database that organises genes and variations affecting haemoglobinopathies,

157 including disease-causing and disease-modifying variations, as well as diagnostically relevant

158 neutral polymorphisms [2]. IthaGenes entries started out with globin gene causative mutations

159 and annotations initially collated in the books "A Syllabus of Human Hemoglobin Variants

160 (Second Edition)" [21] and "A Syllabus of Thalassemia Mutations" [22] and has since

161 comprehensively incorporated any subsequently reported variations and annotations from

162 recent articles and reports [23-26] and from existing information on other public databases,

163 such as HbVar [16], dbSNP [11], ClinVar [13], OMIM [12] and SwissVar [27]. IthaGenes

164 has six main sections covering the catalogue of genes and regions, the catalogue of mutations,

165 advanced search, statistics, the catalogue of references, and, finally, frequently asked 166 questions (FAQs). Advanced search provides a powerful interface for queries and access to 167 all IthaGenes database entries, with a wide range of search options as previously described 
168 [2]. Moreover, IthaGenes integrates the NCBI sequence viewer for detailed graphical

169 representation of each variation and provides phenotype, epidemiology and HPLC data,

170 related publications and external links. Comprehensive coverage, search functionality,

171 extensive annotation and visualisation, systematic inclusion of external links and

172 interconnectedness internally and with other ITHANET portal sections make IthaGenes an

173 exceptional resource for professionals in the field.

174 Over the past few years, IthaGenes has been established as the largest database of variations

175 related to haemoglobinopathies, with the annotation of around 2000 disease-causing or

176 potentially disease-causing variations. Notably, a joint ITHANET-GG2020 application is

177 currently under review by ClinGen for the assignment of an Expert Panel status towards

178 comprehensive annotation of all variants affecting haemoglobinopathies, based on the

179 guidelines of the American College of Medical Genetics and Genomics (ACMG), and their

180 submission to the NCBI ClinVar database [13]. An ongoing pilot study evaluating the Panel's

181 haemoglobinopathy-specific rules for a subset of variations will help fine-tune the

182 classification criteria and represents the final step required for Panel approval by ClinGen.

183 The Panel will begin the variant annotation with the genes located in the globin gene clusters,

184 which carry the commonest and most highly penetrant variations, and will subsequently

185 proceed with annotation of variations located in other genes that have a modifying effect on

186 haemoglobinopathies.

187 The first IthaGenes release [2] was mainly focused on the annotation of variations on the

188 globin gene clusters with a small number of additional modifier genes. However, the

189 enormous and still expanding accumulation of data from genome-wide association studies

190 aiming to identify novel modifiers of haemoglobinopathies has resulted in the accumulation

191 of new genes and loci associated with haemoglobinopathy-related phenotypes. In fact,

192 information on trans-acting modifiers is already considered as an important factor in the

193 clinical management of patients with haemoglobinopathies [28]. Thus and after incorporating

194 a large number of genome-wide or targeted association studies over the past two years, the

195 number of genes and loci annotated in IthaGenes has increased from 32 to a total of 232 and,

196 consequently, the number of annotated variations from 1963 to a total of 2690 , comprising 
197 around 600 disease-modifying variants. In addition, most variants are assigned at least one of

19841 clinical phenotypes included in IthaGenes that are now linked with established phenotypic

199 annotations by the Human Phenotype Ontology [29,30] and OMIM [12]. Figure 2 shows the

200 distribution of disease-modifying mutations by their associated phenotype.

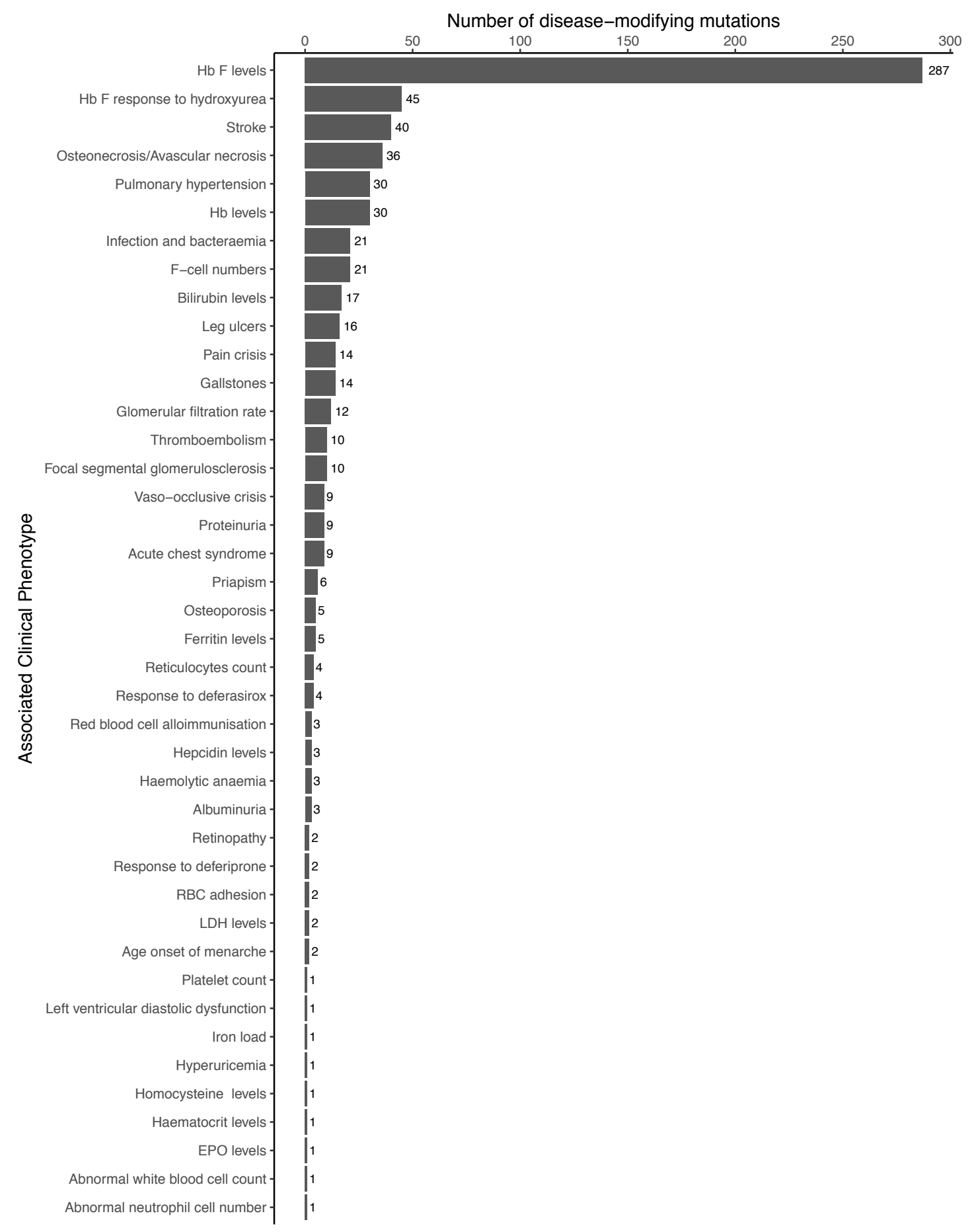

Figure 2: The distribution of disease-modifying mutations by associated clinical 
206 New variations can be directly reported by completing the IthaGenes submission form or by

207 using the ITHANET contact form (http://www.ithanet.eu/contact). Data stored in IthaGenes

208 are freely available for download in CSV and JSON format.

\section{IthaMaps}

210 IthaMaps is a global epidemiology database of haemoglobinopathies, illustrating data on a

211 dynamic global to regional map. For the first release of the IthaMaps database [2],

212 information on relative mutation frequencies were extracted from 89 publications and were

213 illustrated on an interactive map at the country level for a total of 56 countries. Herein, the

214 database and interface have been revamped to allow integration of additional types of

215 information and more detailed data. Specifically, the IthaMaps content can now be divided

216 into three classes: (a) country-specific information on existing haemoglobinopathy-related

217 healthcare policies, (b) country-specific information on the status of major

218 haemoglobinopathies, such as the prevalence, incidence and overall burden of $\beta$-thalassaemia

219 and SCD, and (c) relative allele frequencies of specific globin gene mutations at the national

220 and regional level, dynamically linked to corresponding IthaGenes entries.

221 Table 1: The distribution of annotated countries in IthaMaps for each parameter describing 222 existing haemoglobinopathy-related healthcare policies.

\begin{tabular}{|l|r|r|r|r|}
\hline \multirow{2}{*}{ Healthcare policy } & \multicolumn{3}{|c|}{ Number of countries } \\
\cline { 2 - 5 } & Yes (National) & Yes (Regional) & No & \multicolumn{1}{c|}{ Total } \\
\hline Prevention programme & 28 & 9 & 37 & $\mathbf{7 4}$ \\
SCD Newborn screening & 27 & 8 & 74 & $\mathbf{1 0 9}$ \\
Antenatal screening & 14 & 4 & 14 & $\mathbf{3 2}$ \\
Prenatal screening & 21 & 13 & 31 & $\mathbf{6 5}$ \\
Haemoglobinopathies patient registry & 20 & 5 & 15 & $\mathbf{5 0}$ \\
Rare disease patient registry & 7 & 1 & 15 & $\mathbf{2 3}$ \\
Dedicated treatment centres & 33 & 19 & 6 & $\mathbf{5 8}$ \\
Blood transfusion availability & 41 & 16 & 2 & $\mathbf{5 9}$ \\
Iron chelation availability & 26 & 7 & 2 & $\mathbf{3 5}$ \\
MRI facilities & 17 & 9 & 8 & $\mathbf{3 4}$ \\
Patient associations & 63 & 1 & 2 & $\mathbf{6 6}$ \\
Genetic counselling & 29 & 6 & 4 & $\mathbf{3 9}$ \\
\hline
\end{tabular}

224 In addition and through synergies of ITHANET with the HVP GG2020 Challenge, IthaMaps

225 has benefitted significantly from direct contributions to its content from local experts, with 21

226 scientists annotating or reviewing data for their countries of expertise. In combination with a

227 high volume of extracted information from a total of 287 published reports, IthaMaps

228 organises epidemiological data for 183 countries, thus becoming a valuable tool for 
230 the distribution of annotated countries for parameters that describe existing

231 haemoglobinopathy-related healthcare policies, and Table 2 shows the number of annotated countries per continent for epidemiological parameters that describe the status and burden of major haemoglobinopathies.

Table 2: The distribution of annotated countries in IthaMaps per continent for each parameter describing the status of major haemoglobinopathies.

\begin{tabular}{|l|r|r|r|r|r|r|r|}
\cline { 2 - 7 } \multicolumn{1}{c|}{} & \multicolumn{6}{c|}{ Number of countries } \\
\cline { 2 - 7 } \multicolumn{1}{c|}{} & Africa & Asia & Europe & $\begin{array}{c}\text { North } \\
\text { America }\end{array}$ & $\begin{array}{c}\text { South } \\
\text { America }\end{array}$ & Oceania & Total \\
\hline Prevalence of $\beta$-thalassaemia carriers & 12 & 43 & 31 & 11 & 6 & 2 & $\mathbf{1 0 5}$ \\
Prevalence of SCD carriers & 30 & 18 & 21 & 32 & 10 & - & $\mathbf{1 1 1}$ \\
Prevalence of $\alpha$-thalassaemia carriers & 7 & 27 & 3 & - & 2 & - & $\mathbf{3 9}$ \\
Prevalence of Hb E carriers & - & 20 & 14 & 2 & 1 & 1 & $\mathbf{3 8}$ \\
Prevalence of Hb C carriers & 12 & 2 & 14 & 31 & 6 & - & $\mathbf{6 5}$ \\
Expected incidence of $\beta$-thalassaemia & 6 & 41 & 28 & 9 & 4 & 1 & $\mathbf{8 9}$ \\
Expected incidence of SCD & 7 & 14 & 2 & 10 & 4 & - & $\mathbf{3 7}$ \\
Incidence of $\beta$-thalassaemia & - & 4 & 4 & - & 1 & - & $\mathbf{9}$ \\
Incidence of SCD & 51 & 18 & 20 & 25 & 9 & 1 & $\mathbf{1 2 4}$ \\
Incidence of $\alpha$-thalassaemia & 1 & - & - & - & - & - & $\mathbf{1}$ \\
Known $\beta$-thalassaemia patients & 4 & 32 & 17 & 3 & - & 1 & $\mathbf{5 7}$ \\
Known SCD patients & 3 & 16 & 16 & 4 & 1 & - & $\mathbf{4 0}$ \\
\hline
\end{tabular}

238 In addition, IthaMaps organises information on relative mutation frequencies, at national or 239 regional level, for 66 countries and for a total of 333 globin gene mutations. To facilitate the 240 identification of the most suitable report, IthaMaps provides detailed information about the 241 parameters of each study, including the sample description (e.g. sample size, population 242 description, specific ethnic group), country region/province, study period and related 243 publication, whilst visualisations of mutation frequencies can be filtered based on the above 244 study parameters.

245 All epidemiological parameters stored in IthaMaps can be visualised on a worldwide or 246 country-specific interactive map, which can be exported in a variety of image formats. Figure 2473 shows three examples of possible illustrations available in IthaMaps: (A) existing healthcare 248 policies with regards to newborn screening for SCD, (B) the worldwide distribution of $\beta$ 249 thalassaemia carriers, and (C) the distribution of a selected mutation (e.g. IVS I-5 G $>$ C) in a country (e.g. India) with different frequencies for each province. 


\section{A. Newborn Screening for SCD worldwide}

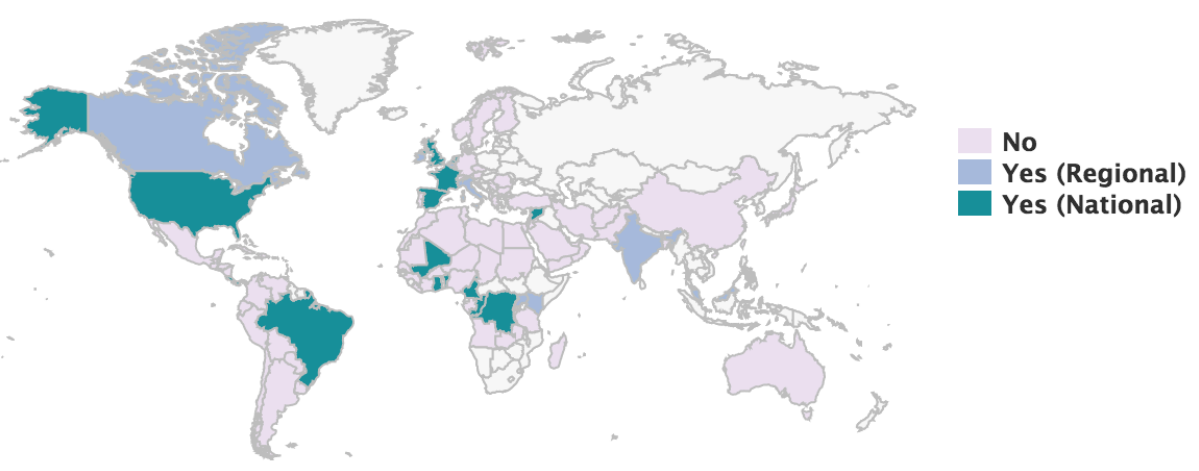

\section{B. Prevalence of $\beta$-thalassaemia carriers worldwide}

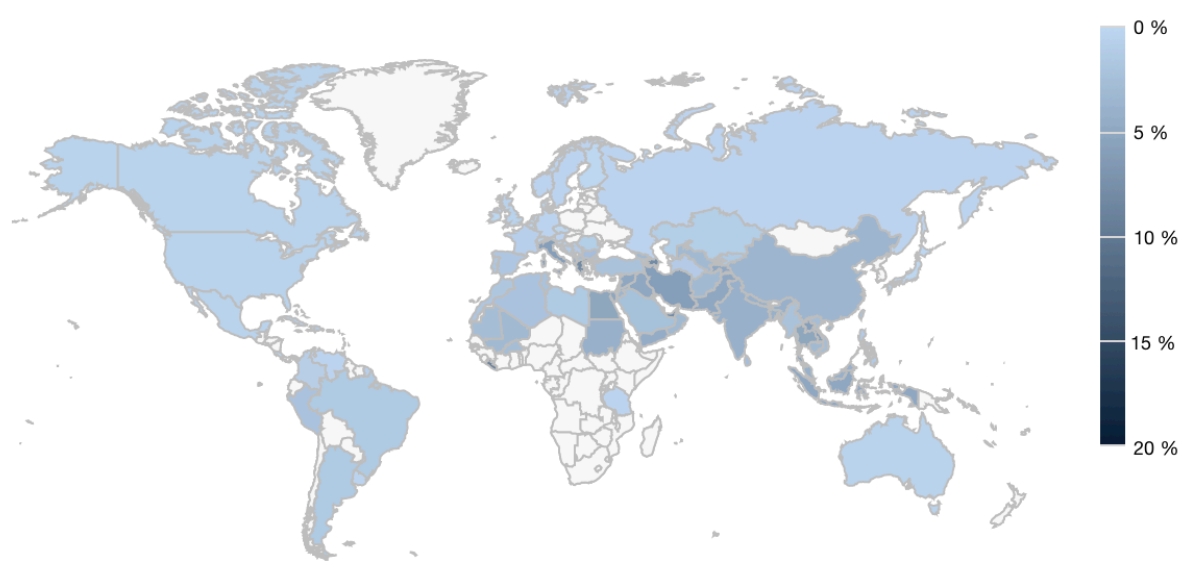

\section{Distribution of IVS I-5 (G>C) in India}
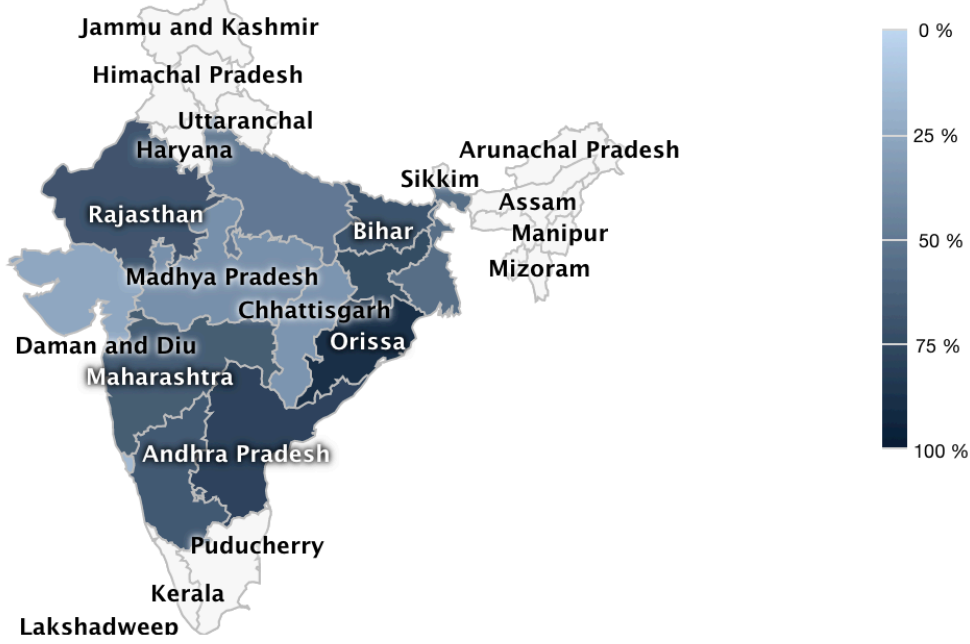

Figure 3: Examples of possible dynamic illustrations available in IthaMaps: (A) existing healthcare policies with regards to prenatal screening, (B) the worldwide distribution of $\beta$-thalassaemia carriers, and $(\mathrm{C})$ the relative frequency distribution of mutation IVS I-5 


\section{IthaChrom}

258 Structural variants of the polypeptide globin chains underlying haemoglobinopathies are most

259 commonly detected with the utilisation of high performance liquid chromatography (HPLC).

260 However, their definitive diagnosis is becoming a challenging task, owing to the large and

261 increasing number of newly identified $\mathrm{Hb}$ variants, with around $1360 \mathrm{Hb}$ variants currently

262 stored in IthaGenes. IthaChrom aims at supporting the diagnosis of structural

263 haemoglobinopathies by providing digitised anonymised reports (as kindly provided by Bio-

264 Rad Laboratories Inc.) of standard diagnostic HPLC analyses. The interface allows database

265 searches of key data, such as the retention time, specific globin genes, Hb variants, HPLC

266 instruments and methodologies. To date, more than 600 HPLC diagnostic reports are

267 available in IthaChrom and we aim at expanding this section by increasing the catalogue of

268 HPLC methodologies and programmes and by allowing contributions from additional

269 laboratories

\section{DISCUSSION}

271 The ITHANET Portal is a growing public resource on haemoglobinopathies and, as an

272 interactive community tool, it invites contributions to its content, including news, events,

273 publications and clinical trials, and contact information for clinical, research and diagnostic

274 centres and patient organisations for haemoglobinopathies as well as epidemiological, genetic

275 and diagnostic data. Through these contributions and centralised development, the ITHANET

276 portal aims at expanding its current content with useful tools for scientists and policymakers

277 involved in researching, diagnosing, preventing and treating haemoglobinopathies.

278 Specifically, future developments of the ITHANET portal include the annotation of IthaGenes

279 entries according to the guidelines by the ACMG [31], as part of the ITHANET-GG2020

280 Expert Panel, the development of epidemiological tools to further facilitate evidence-based

281 policymaking and the implementation of a complete genotype-phenotype database for

282 haemoglobinopathies. Notably, ITHANET is maintained by permanent funding, thus ensuring

283 long-term development and update of the portal, and is an active partner in and beneficiary of

284 primary research data from several projects on haemoglobinopathies. In addition, ITHANET

285 is an active member of the European Reference Network EuroBloodNet for rare 
haematological diseases and is involved in the development of patient registries for the non-

malignant section of the network. Most importantly, through its partnership with the global

HVP GG2020 Challenge, ITHANET has become a comprehensive resource for all

information relating to the haemoglobinopathies and is evolving into an indispensable tool for

the research, prevention and diagnosis of haemoglobinopathies.

\section{ACKNOWLEDGEMENTS}

We are grateful to the following scientists that contributed or curated data: Aurelio Maggio,

Bertha Ibarra, Casten W Lederer, Catherine Badens, Celeste Bento, Coralea Stephanou,

294 Cornelis L Harteveld, Esri Voskaridou, Fahd Al-Mulla, Hafizur Rahman, Hassan Syahzuwan,

295 Jacques Elion, Joanne Traeger-Synodinos, Joan-LLuis Vives Corrons, John Old, Leticia

296 Ribeiro, Léon Tshilolo, Marina Kleanthous, Mas Rina Wati Abdoul Hamid, Michael

297 Angastiniotis, Abiageli Nnodu, Paolo Moi, Pavlos Fanis, Petros Kountouris, Salah Al-

298 Humood, Valeriya Kaleva, Xiangmin Xu, Yvonne Daniel, and Bin Alwi Zilfalil. An up-to-

299 date list of ITHANET contributors and curators is provided at

300 http://www.ithanet.eu/community/ithanet-contributors. The authors would like to thank the

301 Cyprus Institute of Neurology and Genetics for funding and computer equipment and for

302 hosting ITHANET. The authors are also grateful to Marco Flamini and Bio-Rad Laboratories,

303 Inc. for providing the HPLC images and reports.

\section{REFERENCES}

305 1. Modell B, Darlison M. Global epidemiology of haemoglobin disorders and derived service indicators. Bull World Health Organ. 2008;86: 480-487.

307 2. Kountouris P, Lederer CW, Fanis P, Feleki X, Old J, Kleanthous M. IthaGenes: An

308 Interactive Database for Haemoglobin Variations and Epidemiology. PLoS ONE.

309 Public Library of Science; 2014;9: e103020. doi:10.1371/journal.pone.0103020

310 3. Cappellini M-D, Cohen A, Eleftheriou A, Piga A, Porter J, Taher A. Guidelines for the 311 clinical management of thalassaemia. 2nd ed. Nicosia: Thalassaemia International $312 \quad$ Federation; 2008. pp. 1-202.

313 4. Angastiniotis M, Modell B. Global epidemiology of hemoglobin disorders. Ann N Y

5. Kountouris P, Kousiappa I, Papasavva T, Christopoulos G, Pavlou E, Petrou M, et al. retrospective study. Sci Rep. 2016;6: 26371. doi:10.1038/srep26371

318 6. Thein SL. Genetic modifiers of beta-thalassemia. Haematologica. 2005;90: 649-660. 
7. Henderson S, Timbs A, McCarthy J, Gallienne A, Van Mourik M, Masters G, et al.

12. Hamosh A, Scott AF, Amberger J, Valle D, McKusick VA. Online Mendelian Inheritance in Man (OMIM). Hum Mutat. 2000;15: 57-61. doi:10.1002/(SICI)10981004(200001)15:1<57::AID-HUMU12>3.0.CO;2-G

13. Landrum MJ, Lee JM, Riley GR, Jang W, Rubinstein WS, Church DM, et al. ClinVar: public archive of relationships among sequence variation and human phenotype. Nucleic Acids Res. 2014;42: D980-5. doi:10.1093/nar/gkt1113

14. Cooper DN, Ball EV, Krawczak M. The human gene mutation database. Nucleic Acids Res. 1998;26: 285-287. doi:10.1093/nar/26.1.285

15. Johnston JJ, Biesecker LG. Databases of genomic variation and phenotypes: existing resources and future needs. Human Molecular Genetics. 2013;22: R27-31. doi: $10.1093 / \mathrm{hmg} / \mathrm{ddt} 384$

16. Hardison RC, Chui DHK, Giardine B, Riemer C, Patrinos GP, Anagnou N, et al. HbVar: A relational database of human hemoglobin variants and thalassemia mutations at the globin gene server. Hum Mutat. 2002;19: 225-233. doi:10.1002/humu. 10044

17. Fokkema IFAC, Taschner PEM, Schaafsma GCP, Celli J, Laros JFJ, Dunnen den JT. LOVD v.2.0: the next generation in gene variant databases. Hum Mutat. 2011;32: 557-563. doi:10.1002/humu. 21438

18. Giardine B, Borg J, Higgs DR, Peterson KR, Philipsen S, Maglott D, et al. Systematic documentation and analysis of human genetic variation in hemoglobinopathies using the microattribution approach. Nature Genetics. 2011;43: 295-301. doi:10.1038/ng.785

19. Lederer CW, Basak AN, Aydinok Y, Christou S, El-Beshlawy A, Eleftheriou A, et al. An electronic infrastructure for research and treatment of the thalassemias and other hemoglobinopathies: the Euro-mediterranean ITHANET project. Hemoglobin. 2009;33: 163-176. doi:10.1080/03630260903089177

20. Robinson HM. Increasing the involvement of diverse populations in genomics-based health care-lessons from haemoglobinopathies. Journal of community genetics. Springer Berlin Heidelberg; 2017;43: 295-8. doi:10.1007/s12687-017-0327-3

362 21. Huisman THJ, Carver MFH, Efremov GD. A Syllabus of Human Hemoglobin 
22. Huisman THJ, Carver MFH, Baysal E. A Syllabus of Thalassemia Mutations. Augusta, GA, USA: The Sickle Cell Anemia Foundation; 1997.

23. Steinberg MH, Forget BG, Higgs DR, Weatherall DJ. Disorders of Hemoglobin. 2nd ed. Cambridge University Press; 2009.

24. Old J, Angastiniotis M, Eleftheriou A, Galanello R, Harteveld CL, Petrou M, et al. Prevention of Thalassaemias and Other Haemoglobinopathies. 2nd ed. Nicosia, Cyprus: Thalassaemia International Federation; 2013.

25. Thein SL. The molecular basis of $\beta$-thalassemia. Cold Spring Harbor Perspectives in Medicine. 2013;3: a011700. doi:10.1101/cshperspect.a011700

26. Higgs DR. The molecular basis of $\alpha$-thalassemia. Cold Spring Harbor Perspectives in Medicine. 2013;3: a011718. doi:10.1101/cshperspect.a011718

27. Mottaz A, David FPA, Veuthey A-L, Yip YL. Easy retrieval of single amino-acid polymorphisms and phenotype information using SwissVar. Bioinformatics. 2010;26: 851-852. doi:10.1093/bioinformatics/btq028

28. Danjou F, Anni F, Perseu L, Satta S, Dessì C, Lai ME, et al. Genetic modifiers of $\beta$ thalassemia and clinical severity as assessed by age at first transfusion. Haematologica. Haematologica; 2012;97: 989-993. doi:10.3324/haematol.2011.053504

29. Robinson PN, Köhler S, Bauer S, Seelow D, Horn D, Mundlos S. The Human Phenotype Ontology: A Tool for Annotating and Analyzing Human Hereditary Disease. The American Journal of Human Genetics. Elsevier; 2008;83: 610-615. doi:10.1016/j.ajhg.2008.09.017

30. Köhler S, Doelken SC, Mungall CJ, Bauer S, Firth HV, Bailleul-Forestier I, et al. The Human Phenotype Ontology project: linking molecular biology and disease through phenotype data. Nucleic Acids Res. 2014;42: D966-74. doi:10.1093/nar/gkt1026

31. Richards S, Aziz N, Bale S, Bick D, Das S, Gastier-Foster J, et al. Standards and guidelines for the interpretation of sequence variants: a joint consensus recommendation of the American College of Medical Genetics and Genomics and the Association for Molecular Pathology. Genet Med. Nature Publishing Group; 2015. doi:10.1038/gim.2015.30 